ANNALES

POLONICI MATHEMATICI

LXXVIII.3 (2002)

\title{
On local-in-time existence for the Dirichlet problem for equations of compressible viscous fluids
}

\author{
by Piotr Boguseaw Mucha and \\ WOJCIECH ZAJĄCZKOWSKI (Warszawa)
}

\begin{abstract}
The local existence of solutions for the compressible Navier-Stokes equations with the Dirichlet boundary conditions in the $L_{p}$-framework is proved. Next an almost-global-in-time existence of small solutions is shown. The considerations are made in Lagrangian coordinates. The result is sharp in the $L_{p}$-approach, because the velocity belongs to $W_{r}^{2,1}$ with $r>3$.
\end{abstract}

1. Introduction. In this paper we consider the motion of viscous compressible fluids in a bounded domain $\Omega \subset \mathbb{R}^{3}$ described by the Navier-Stokes equations

$$
\begin{array}{ll}
\varrho\left(v_{t}+v \cdot \nabla v\right)+\mu \Delta v-\nu \nabla \operatorname{div} v+\nabla p=\varrho f & \text { in } \Omega^{T}, \\
\varrho_{t}+\operatorname{div}(\varrho v)=0 & \text { in } \Omega^{T}, \\
v=\alpha & \text { on } S^{T}, \\
\left.v\right|_{t=0}=v_{0},\left.\quad \varrho\right|_{t=0}=\varrho_{0} & \text { in } \Omega,
\end{array}
$$

where $\Omega^{T}=\Omega \times[0, T], S=\partial \Omega, S^{T}=S \times[0, T], v(x, t)$ is the velocity of the fluid, $\varrho(x, t)$ the density, $p=p(\varrho)$ the pressure, $f(x, t)$ the external force, $\mu$ and $\nu$ the constant viscosity coefficients which satisfy the thermodynamic restrictions

$$
\mu>0, \quad \nu>\frac{1}{3} \mu
$$

and the dot denotes the scalar product in $\mathbb{R}^{3}$.

To prove the existence of solutions to (1.1) we introduce the Lagrangian coordinates as the initial data for the Cauchy problem

$$
\frac{d x}{d t}=v(x, t),\left.\quad x\right|_{t=0}=\xi \in \Omega .
$$

2000 Mathematics Subject Classification: 35Q30, 76Q10.

Key words and phrases: local existence, sharp regularity, almost global solutions.

Research supported by Polish KBN Grant 2 P03A 03816. 
Solving (1.3) we obtain

$$
x=\xi+\int_{0}^{t} u(\xi, \tau) d \tau \equiv x(\xi, t)
$$

which is the relation between the Eulerian $x$ and Lagrangian $\xi$ coordinates and $u(\xi, t)=v(x(\xi, t), t)$. Here we need to add an extra condition $\left.v \cdot \bar{n}\right|_{S^{T}}=0$ $(\bar{n}$ is the normal vector to $S$ ) which ensures that this transformation preserves the domain $(\{x \mapsto \xi\}: \Omega \rightarrow \Omega)$.

In Lagrangian coordinates, (1.1) reads

$$
\begin{array}{ll}
\eta u_{t}-\mu \Delta_{u} u-\nu \nabla_{u} \operatorname{div}_{u} u+\nabla_{u} q=\eta g & \text { in } \Omega^{T}, \\
\eta_{t}+\eta \operatorname{div}_{u} u=0 & \text { in } \Omega^{T}, \\
u=\alpha & \text { on } S^{T}, \\
\left.u\right|_{t=0}=v_{0},\left.\quad \eta\right|_{t=0}=\varrho_{0} & \text { in } \Omega,
\end{array}
$$

where $\eta(\xi, t)=\varrho(x(\xi, t), t), q=p(\eta), g(\xi, t)=f(x(\xi, t), t), \nabla_{u}=\frac{\partial \xi_{i}}{\partial x} \partial_{\xi_{i}}$, $\Delta_{u}=\nabla_{u}^{2}, \operatorname{div}_{u}=\nabla_{u}$. and the summation convention over repeated indices is used.

The first result of the paper is the following.

Theorem 1.1. Let $r>3, S \in W_{r}^{2-1 / r}, f \in L_{r}\left(0, T ; W_{\infty}^{1}(\Omega)\right), \alpha \in$ $C^{3}\left(S^{T}\right), v_{0} \in W_{r}^{2-2 / r}(\Omega), \varrho_{0} \in W_{r}^{1}(\Omega), \alpha \cdot \bar{n}=0$ and moreover

$$
0<2 a \leq \varrho_{0}(x) \leq b<\infty .
$$

Then there exists $T_{0}>0$ such that for $T \leq T_{0}$ there exists a unique solution of (1.5) such that $u \in W_{r}^{2,1}\left(\Omega^{T}\right), \eta \in W_{r}^{1,0}\left(\Omega^{T}\right), \eta_{t} \in W_{r}^{1,0}\left(\Omega^{T}\right)$ and

$$
\begin{aligned}
& \|u\|_{W_{r}^{2,1}\left(\Omega^{T}\right)}+\|\eta\|_{W_{r}^{1,0}\left(\Omega^{T}\right)}+\left\|\eta_{t}\right\|_{W_{r}^{1,0}\left(\Omega^{T}\right)} \\
& \leq c\left(\|f\|_{L_{r}\left(\Omega^{T}\right)}+\|\alpha\|_{W_{r}^{2-1 / r, 1-1(2 r)}\left(S^{T}\right)}+\left\|v_{0}\right\|_{W_{r}^{2-2 / r}(\Omega)}+\left\|\varrho_{0}\right\|_{W_{r}^{1}(\Omega)}\right. \\
& \left.+\|f\|_{L_{r}\left(0, T ; W_{\infty}^{1}(\Omega)\right)}+\|\alpha\|_{C^{3}\left(S^{T}\right)}\right)
\end{aligned}
$$

and

$$
0<a \leq \eta(x, t) \leq 2 b<\infty
$$

for $(x, t) \in \Omega^{T}$.

The next result concerns the time continuity of solutions which are close to equilibrium states

$$
\nabla p(\bar{\varrho}(x))=\bar{\varrho}(x) f(x)
$$

where

$$
0<\bar{\varrho}_{*} \leq \bar{\varrho}(x) \leq \bar{\varrho}^{*}<\infty
$$

for each $x \in \Omega, f=\nabla \varphi$ and $\bar{\varrho}_{*}, \bar{\varrho}^{*}$ are given constants. 
From (1.1) with $\alpha=0, f=\nabla \varphi$ and (1.8) we obtain the following system for perturbations in Eulerian coordinates:

$$
\begin{array}{ll}
\varrho\left(v_{t}+v \cdot \nabla v\right)+\mu \Delta v-\nu \nabla \operatorname{div} v+\gamma \nabla \sigma=\sigma f-\sigma \nabla \gamma & \text { in } \Omega^{T}, \\
\sigma_{t}+\operatorname{div}(\varrho v)=0 & \text { in } \Omega^{T}, \\
v=0 & \text { on } S^{T}, \\
\left.v\right|_{t=0}=v_{0},\left.\quad \sigma\right|_{t=0}=\varrho_{0}-\bar{\varrho} & \text { in } \Omega,
\end{array}
$$

where

$$
\sigma(x, t)=\varrho(x, t)-\bar{\varrho}(x)
$$

and $\gamma$ is defined by the relation

$$
p(\varrho)-p(\bar{\varrho})=\sigma \int_{0}^{1} p^{\prime}(\varrho+s(\bar{\varrho}-\varrho)) d s=\sigma \gamma .
$$

System (1.9) in Lagrangian coordinates reads

$$
\begin{array}{ll}
\eta u_{t}-\mu \Delta_{u} u-\nu \nabla_{u} \operatorname{div}_{u} u+\gamma \nabla_{u} \chi=\chi g-\chi \nabla_{u} \gamma & \text { in } \Omega^{T}, \\
\chi_{t}+\eta \operatorname{div}_{u} u=-\bar{\eta}_{t} & \text { in } \Omega^{T}, \\
u=0 & \text { on } S^{T}, \\
\left.u\right|_{t=0}=v_{0},\left.\quad \eta\right|_{t=0}=\varrho_{0}-\bar{\varrho} & \text { in } \Omega,
\end{array}
$$

where

$$
\chi(\xi, t)=\eta(\xi, t)-\bar{\eta}(\xi, t)
$$

and $\bar{\eta}(\xi, t)=\bar{\varrho}(x(\xi, t))$.

We prove the almost-global-in-time existence of solutions to (1.10).

Theorem 1.2. Let $r>3, f=\nabla \varphi \in W_{\infty}^{1}(\Omega), \varrho_{0}-\bar{\varrho} \in W_{r}^{1}(\Omega), u_{0} \in$ $W_{r}^{2-2 / r}(\Omega), p(\varrho)=a \varrho^{\kappa}$ with $a>0, \kappa>1$ and $\varrho_{0} \geq \bar{\varrho}_{*} / 2$. Let $T>0$ be given. Then there exist $\bar{M}_{1}(T)$ and $M_{2}\left(T, M_{1}\right)$, where $M_{2}\left(T, M_{1}\right) \rightarrow 0$ as $T \rightarrow \infty$ and $M_{1} \rightarrow 0$, such that for $M_{1} \leq \bar{M}_{1}(T)$, if

$$
\left\|v_{0}\right\|_{W_{r}^{2-2 / r}(\Omega)}+\left\|\varrho_{0}-\bar{\varrho}\right\|_{W_{r}^{1}(\Omega)} \leq M_{2}\left(T, M_{1}\right),
$$

then there exist solutions of (1.10) such that $u \in W_{r}^{2,1}(\Omega \times[0, T]), \chi \in$ $V_{r}(\Omega \times[0, T])$ and

$$
\|u\|_{W_{r}^{2,1}(\Omega \times[0, T])}+\|\eta\|_{V_{r}(\Omega \times[0, T])} \leq M_{1} .
$$

Moreover,

$$
\eta(\xi, t) \geq \bar{\varrho}_{*} / 4
$$

for each $(\xi, t) \in \Omega \times[0, T]$. 
To prove these results we need solvability of the following linear problem in the $L_{r}$-framework:

$$
\begin{aligned}
& C u_{t}-\mu \Delta u-\nu \nabla \operatorname{div} u+A \nabla \eta=F, \\
& \eta_{t}+B \operatorname{div} u=H, \\
& \left.u\right|_{S_{T}}=G, \\
& \left.u\right|_{t=0}=u_{0},\left.\quad \eta\right|_{t=0}=\eta_{0},
\end{aligned}
$$

where $A(x, t), B(x, t), C(x, t)$ are positive functions which belong to $C^{\alpha}(\Omega \times$ $[0, T])$.

For system (1.11) we have proved the following result (see [3]).

TheOREm 1.3. Let $r>3, S \in W_{r}^{2-1 / r}, F \in L_{r}\left(\Omega_{T}\right), H \in W_{r}^{1,0}\left(\Omega_{T}\right)$, $G \in W_{r}^{2-1 / r, 1-1 /(2 r)}\left(S_{T}\right), u_{0} \in W_{r}^{2-2 / r}(\Omega), \eta_{0} \in W_{r}^{1}(\Omega)$ and

$$
0<C_{*} \leq A(x, t), B(x, t), C(x, t) \leq C^{*}<\infty \text {. }
$$

Moreover, A, B, C belong to $C^{\alpha}\left(\Omega^{T}\right), \alpha>0$. Then there exists a unique solution of problem (1.11) such that $u \in W_{r}^{2,1}\left(\Omega^{T}\right), \eta \in W_{r}^{1,0}\left(\Omega^{T}\right), \eta_{t} \in$ $W_{r}^{1,0}\left(\Omega^{T}\right)$ and

$$
\begin{array}{r}
\|u\|_{W_{r}^{2,1}\left(\Omega^{T}\right)}+\|\eta\|_{W_{r}^{1,0}\left(\Omega^{T}\right)}+\left\|\eta_{t}\right\|_{W_{r}^{1,0}\left(\Omega^{T}\right)} \\
\leq c\left(T, C^{*}, C_{*}\right)\left[\|f\|_{L_{r}\left(\Omega^{T}\right)}+\|H\|_{W_{r}^{1,0}\left(\Omega^{T}\right)}+\|G\|_{W_{r}^{2-1 / r, 1-1(2 r)}\left(S^{T}\right)}\right. \\
\left.+\left\|u_{0}\right\|_{W_{r}^{2-2 / r}(\Omega)}+\left\|\eta_{0}\right\|_{W_{r}^{1,0}(\Omega)}\right]
\end{array}
$$

where $c(T, \cdot, \cdot)$ is an increasing function of $T$.

The global existence of small regular solutions for problem (1.9) has been shown in $[2,5]$ for the case when the norms of $\varphi(f=\nabla \varphi)$ are sufficiently small. These results base on energy estimates. In our paper we show existence and almost-global-in-time existence of regular solutions in the $L_{p}$-framework. The theorems enable us to prove in [4] the global existence of small solutions to problem (1.9) with "large" $\varphi$. Moreover this result is sharp in the $L_{p}$-framework.

2. Notation. In our considerations we will need the anisotropic SobolevSlobodetskil spaces $W_{r}^{m, n}\left(Q^{T}\right)$ where $m, n \in \mathbb{R}_{+} \cup\{0\}, r \geq 1$ and $Q^{T}=$ $Q \times(0, T)$ with the norm

$$
\begin{aligned}
\|u\|_{W_{r}^{m, n}\left(Q^{T}\right)}^{r}= & \int_{0}^{T} \int_{Q}|u(x, t)|^{r} d x d t \\
& +\sum_{0 \leq\left|m^{\prime}\right| \leq[|m|]} \int_{0}^{T} \int_{Q}\left|D_{x}^{m^{\prime}} u(x, t)\right|^{r} d x d t
\end{aligned}
$$




$$
\begin{aligned}
& +\sum_{\left|m^{\prime}\right|=[|m|]} \int_{0}^{T} d t \iint_{Q} \frac{\left|D_{x}^{m^{\prime}} u(x, t)-D_{x^{\prime}}^{m^{\prime}} u\left(x^{\prime}, t\right)\right|^{r}}{\left|x-x^{\prime}\right| s+r(|m|-[|m|])} d x d x \\
& +\sum_{0 \leq\left|n^{\prime}\right| \leq[|n|]} \int_{0}^{T} \int_{Q}^{T}\left|D_{t}^{n^{\prime}} u(x, t)\right|^{r} d x d t \\
& +\int_{Q} d x \int_{0}^{T} \int_{0}^{T} \frac{\left|D_{t}^{[n]} u(x, t)-D_{t^{\prime}}^{[n]}\left(x, t^{\prime}\right)\right|^{r}}{\left|t-t^{\prime}\right|^{1+r(n-[n])}} d t d t^{\prime} .
\end{aligned}
$$

where $s=\operatorname{dim} Q,[\alpha]$ is the integral part of $\alpha, D_{x}^{l}=\partial_{x_{1}}^{l_{1}} \ldots \partial_{x_{s}}^{l_{s}}$, where $l=\left(l_{1}, \ldots, l_{s}\right)$ is a multiindex and $|l|=l_{1}+\ldots+l_{s}, l_{i} \geq 0, i=1, \ldots, s$. For $m$ and $n$ integers, the corresponding terms with differences vanish.

We define the space $V\left(\Omega^{T}\right)$ as the closure

$$
V\left(\Omega^{T}\right)=\overline{C^{\infty}\left(\Omega^{T}\right)}\|\cdot\|_{V\left(\Omega^{T}\right)},
$$

where

$$
\|f\|_{V\left(\Omega^{T}\right)}=\|f\|_{W_{r}^{1,0}\left(\Omega^{T}\right)}+\left\|f_{t}\right\|_{W_{r}^{1,0}\left(\Omega^{T}\right)} .
$$

In the proof we will use the following results.

Proposition 2.1 (see [1]). Let $u \in W_{r}^{m, n}\left(\Omega^{T}\right), m, n \in \mathbb{R}_{+}$. If

$$
\kappa=\sum_{i=1}^{3}\left(\alpha_{i}+\frac{1}{r}-\frac{1}{q}\right) \frac{1}{m}+\left(\beta+\frac{1}{r}-\frac{1}{q}\right) \frac{1}{n}<1
$$

then

$$
\left\|D_{t}^{\beta} D_{x}^{\alpha} u\right\|_{L_{q}\left(\Omega^{T}\right)} \leq \varepsilon\|u\|_{W_{r}^{m, n}\left(\Omega^{T}\right)}+c(\varepsilon)\|u\|_{L_{2}\left(\Omega^{T}\right)},
$$

where $q \geq r \geq 2, \varepsilon \in(0,1)$ and $c(\varepsilon) \rightarrow \infty$ as $\varepsilon \rightarrow 0$.

Proposition 2.2. Let $r>3$. Then $V\left(\Omega^{T}\right) \subset C^{\alpha}\left(\Omega^{T}\right)$, where $0<\alpha<$ $1-3 / r$, and

$$
\|f\|_{C^{\alpha}\left(\Omega^{T}\right)} \leq c\|f\|_{V\left(\Omega^{T}\right)} .
$$

Proof. We have $r>3,0<\alpha<1-3 / r$ and the following imbeddings:

$$
\begin{aligned}
f & \in L_{r}\left(0, T ; W_{r}^{1}(\Omega)\right) \subset L_{r}\left(0, T ; C^{\alpha}(\Omega)\right), \\
f_{t} & \in L_{r}\left(0, T ; W_{r}^{1}(\Omega)\right) \subset L_{r}\left(0, T ; C^{\alpha}(\Omega)\right) .
\end{aligned}
$$

From these two relations we conclude

$$
f \in W_{r}^{1}\left(0, T ; C^{\alpha}(\Omega)\right) \subset C^{\alpha^{\prime}}\left(0, T ; C^{\alpha}(\Omega)\right),
$$

where $0<\alpha^{\prime}<1-1 / r$. Thus we obtain $f \in C^{\alpha}\left(\Omega^{T}\right)$.

In our considerations we will treat all imbedding theorems for Sobolev spaces as well known results. All constants are denoted by $c$. 
3. Proof of Theorem 1.1. We prove the existence of solutions of (1.5) by the method of successive approximations. We construct a sequence $\left\{u_{m}, \eta_{m}\right\}_{m=0}^{\infty}$ by the relations

$$
\begin{array}{ll}
\quad \eta_{m} u_{m+1, t}-\mu \Delta_{u_{m}} u_{m+1}-\nu \nabla_{u_{m}} \operatorname{div}_{u_{m}} u_{m+1} & \\
\quad+p^{\prime}\left(\eta_{m}\right) \nabla_{u_{m}} \eta_{m+1}=\eta_{m} f\left(x_{m}, t\right) & \text { in } \Omega^{T}, \\
\eta_{m+1, t}+\eta_{m} \operatorname{div}_{u_{m}} u_{m+1}=0 & \text { in } \Omega^{T}, \\
u_{m+1}=\alpha\left(x_{m}, t\right) & \text { on } S^{T}, \\
\left.u_{m+1}\right|_{t=0}=v_{0},\left.\quad \eta_{m+1}\right|_{t=0}=\varrho_{0} & \text { in } \Omega,
\end{array}
$$

where $x_{m}=\xi+\int_{0}^{t} u_{m}\left(x, t^{\prime}\right) d t^{\prime}$ and $u_{0}, \eta_{0}$ are defined by

$$
\begin{array}{ll}
\varrho_{0} u_{0, t}-\mu \Delta u_{0}-\nu \nabla \operatorname{div} u_{0}+p^{\prime}\left(\varrho_{0}\right) \nabla \eta_{0}=\varrho_{0} f(\xi, t) & \text { in } \Omega^{T}, \\
\eta_{0, t}+\varrho_{0} \operatorname{div} u_{0}=0 & \text { in } \Omega^{T}, \\
u_{0}=\alpha(x(\xi, t), t) & \text { on } S^{T}, \\
\left.u_{0}\right|_{t=0}=v_{0},\left.\quad \eta_{0}\right|_{t=0}=\varrho_{0} & \text { in } \Omega .
\end{array}
$$

From Theorem 1.3 we get a solution of (3.2) with the estimate

$$
\leq c(T)\left(\|f\|_{L_{r}\left(\Omega^{T}\right)}+\|\alpha\|_{W_{r}^{2-1 / r, 1-1 /(2 r)}\left(S^{T}\right)}+\left\|v_{0}\right\|_{W_{r}^{2-2 / r}(\Omega)}+\left\|\varrho_{0}\right\|_{W_{r}^{1}(\Omega)}\right) .
$$

First we show that $\left\{u_{m}, \eta_{m}\right\}_{m=0}^{\infty}$ is uniformly bounded on $\Omega^{T}$ for some $T>0$. By induction we show that

$$
X_{k}=\left\|u_{k}\right\|_{W_{r}^{2,1}\left(\Omega^{T}\right)}+\left\|\eta_{k}\right\|_{V\left(\Omega^{T}\right)} \leq 4 M,
$$

where

$$
M=c(T)\left(\|f\|_{L_{r}\left(\Omega^{T}\right)}+\|\alpha\|_{W_{r}^{2-1 / r, 1-1 /(2 r)}\left(S^{T}\right)}+\left\|v_{0}\right\|_{W_{r}^{2-2 / r}(\Omega)}+\left\|\varrho_{0}\right\|_{W_{r}^{1}(\Omega)}\right) .
$$

For fixed $4 M$ we can define $T$ so small that the Jacobian of the transformation (1.4) (with $u_{m}$ ) is bounded.

System (3.1) is examined in the form

$$
\begin{array}{ll}
\eta_{m} u_{m+1, t}-\mu \Delta u_{m+1}-\nu \nabla \operatorname{div} u_{m+1}+p^{\prime}\left(\eta_{m}\right) \nabla \eta_{m+1} & \\
\quad=\eta_{m} f\left(x_{m}, t\right)+p^{\prime}\left(\eta_{m}\right)\left(\nabla-\nabla_{u_{m}}\right) \eta_{m+1} & \\
\quad+\mu\left(\Delta_{u_{m}}-\Delta\right) u_{m+1}+\nu\left(\nabla_{u_{m}} \operatorname{div}_{u_{m}}-\nabla \operatorname{div}\right) u_{m+1} \equiv K & \text { in } \Omega^{T}, \\
\eta_{m+1, t}+\eta_{m} \operatorname{div} u_{m+1}=\eta_{m}\left(\operatorname{div}-\operatorname{div}_{u_{m}}\right) u_{m+1} \equiv L & \text { in } \Omega^{T}, \\
u_{m+1}=\alpha\left(x_{m}, t\right), & \text { on } S^{T}, \\
\left.u_{m+1}\right|_{t=0}=v_{0},\left.\quad \eta_{m+1}\right|_{t=0} & \text { in } \Omega .
\end{array}
$$


By Proposition 2.2 we see that $\eta_{m}, p^{\prime}\left(\eta_{m}\right) \in C^{\alpha}\left(\Omega^{T}\right)$, thus we can apply Theorem 1.3 with $T=1, C_{*}=a$ and $C^{*}=2 b$, to get

$$
\begin{aligned}
\left\|u_{m+1}\right\|_{W_{r}^{2,1}\left(\Omega^{T}\right)}+\left\|\eta_{m+1}\right\|_{V\left(\Omega^{T}\right)} & \\
\leq c_{0}\left(\|f\|_{L_{r}\left(\Omega^{T}\right)}+\|\alpha\|_{W_{r}^{2-1 / r, 1-1 /(2 r)}\left(S^{T}\right)}\right. & +\left\|v_{0}\right\|_{W_{r}^{2-2 / r}(\Omega)}+\left\|\varrho_{0}\right\|_{W_{r}^{1}\left(\Omega^{T}\right)} \\
& \left.+\|K\|_{L_{r}\left(\Omega^{T}\right)}+\|L\|_{W_{r}^{1,0}\left(\Omega^{T}\right)}\right) .
\end{aligned}
$$

To obtain (3.5) we have used the following estimates:

$$
\begin{aligned}
\left\|\alpha\left(x_{m}(\xi, t), t\right)\right\|_{L_{r}\left(S ; W_{r}^{1}(0, T)\right)} & \\
\leq & \left(\int_{S^{T}}\left(|\alpha|^{r}+\left|\alpha_{x_{m}}\right|^{r}\left|u_{m}\right|^{r}+\left|\alpha_{t}\right|^{r}\right)\left|\xi_{x_{m}}\right| d x_{m} d t\right)^{1 / r} \\
& \leq \phi\left(a_{m}\right)\left(\int_{S^{T}}\left(|\alpha|^{r}+\left|\alpha_{x_{m}}\right|^{r}\left|u_{m}\right|^{r}+\left|\alpha_{t}\right|^{r}\right) d x_{m} d t\right)^{1 / r},
\end{aligned}
$$

where $\phi\left(a_{m}\right)$ is a positive increasing function such that $\phi(0)>0$ and $a_{m}=$ $T^{(r-1) / r}\left\|u_{m}\right\|_{W_{r}^{2,1}\left(\Omega^{T}\right)}$. To finish estimating the above term we note

$$
\begin{aligned}
\left(\int_{S^{T}}\left|\alpha_{x}\right|^{r}|u|^{r} d x d t\right)^{1 / r} \leq\left(\int_{0}^{T}\left|\alpha_{x}\right|_{L_{\infty}(S)}^{r} d t\right)^{1 / r}\left(\sup _{t} \int_{S}|u|^{r} d x\right)^{1 / r} \\
\leq\left(\int_{0}^{T}\|\alpha\|_{W_{r}^{2}(S)}^{r} d t\right)^{1 / r}\left(\int_{S} d x\left|\int_{0}^{T} u_{t} d t+v_{0}(x)\right|^{r}\right)^{1 / r} \\
\leq\|\alpha\|_{W_{r}^{2,1}\left(S^{T}\right)}\left(T^{(r-1) / r}\left\|u_{m}\right\|_{W_{r}^{2,1}\left(\Omega^{T}\right)}+\left\|v_{0}\right\|_{W_{r}^{2-2 / r}(\Omega)}\right) .
\end{aligned}
$$

But

$$
\begin{aligned}
K= & \eta_{m} f\left(x_{m}, t\right)+p^{\prime}\left(\eta_{m}\right)\left(\nabla-\nabla_{u_{m}}\right) \eta_{m+1}+\mu\left(\Delta_{u_{m}}-\Delta\right) u_{m+1} \\
& -\nu\left(\nabla \operatorname{div}-\nabla_{u_{m}} \operatorname{div}_{u_{m}}\right) u_{m+1} \\
= & K_{1}+K_{2}+K_{3}+K_{4} .
\end{aligned}
$$

Since $f \in L_{r}\left(0, T ; L_{r}(\Omega)\right)$ we have

$$
\begin{aligned}
\left\|K_{1}\right\|_{L_{r}\left(\Omega^{T}\right)} & =\left\|\eta_{m} f\left(x_{m}, t\right)\right\|_{L_{r}\left(\Omega^{T}\right)} \leq\left\|\eta_{m}\right\|_{L_{\infty}\left(\Omega^{T}\right)}\left\|f\left(x_{m}(\xi, t), t\right)\right\|_{L_{r}\left(\Omega^{T}\right)} \\
& \leq\left\|\eta_{m}\right\|_{L_{\infty}\left(0, T ; W_{r}^{1}(\Omega)\right)} \phi\left(a_{m}\right)\|f\|_{L_{r}\left(\Omega^{T}\right)}
\end{aligned}
$$

and

$$
\begin{aligned}
\left\|\eta_{m}\right\|_{L_{\infty}\left(0, T ; W_{r}^{1}(\Omega)\right)} & \leq\left\|\int_{0}^{T} \eta_{m, t}(t) d t+\varrho_{0}\right\|_{W_{r}^{1}(\Omega)} \\
& \leq T^{(r-1) / r}\left\|\eta_{m}\right\|_{V\left(\Omega^{T}\right)}+\left\|\varrho_{0}\right\|_{W_{r}^{1}(\Omega)}
\end{aligned}
$$


Because $r>3$, by Proposition 2.1 and the Hölder inequality we have

$$
\left\|\int_{0}^{t}\left|\nabla u_{m}\left(x, t^{\prime}\right)\right| d t^{\prime}\right\|_{L_{\infty}\left(\Omega^{T}\right)} \leq c T^{(r-1) / r}\left\|u_{m}\right\|_{W_{r}^{2,1}\left(\Omega^{T}\right)},
$$

which gives

$$
\begin{gathered}
\left\|K_{2}\right\|_{L_{r}\left(\Omega^{T}\right)}=\left\|p^{\prime}\left(\eta_{m}\right)\left(\nabla-\nabla_{u_{m}}\right) \eta_{m+1}\right\|_{L_{r}\left(\Omega^{T}\right)} \\
\leq c T^{(r-1) / r}\left\|u_{m}\right\|_{W_{r}^{2,1}\left(\Omega^{T}\right)}\left\|\eta_{m+1}\right\|_{V\left(\Omega^{T}\right)}, \\
\left\|K_{3}\right\|_{L_{r}\left(\Omega^{T}\right)}=\left\|\mu\left(\Delta_{u_{m}}-\Delta\right) u_{m+1}\right\|_{L_{r}\left(\Omega^{T}\right)} \\
\leq\left\|\mu \operatorname{div}\left(\nabla-\nabla_{u_{m}}\right) u_{m+1}\right\|_{L_{r}\left(\Omega^{T}\right)}+\left\|\mu\left(\operatorname{div}-\operatorname{div}_{u_{m}}\right) \nabla_{u_{m}} u_{m+1}\right\|_{L_{r}\left(\Omega^{T}\right)} \\
\leq c T^{(r-1) / r}\left\|u_{m}\right\|_{W_{r}^{2,1}\left(\Omega^{T}\right)}\left(1+T^{(r-1) / r}\left\|u_{m}\right\|_{W_{r}^{2,1}\left(\Omega^{T}\right)}\right)\left\|u_{m+1}\right\|_{W_{r}^{2,1}\left(\Omega^{T}\right)}
\end{gathered}
$$

and the same for $K_{4}$ :

$$
\begin{aligned}
\left\|K_{4}\right\|_{L_{r}\left(\Omega^{T}\right)} \leq & c T^{(r-1) / r}\left\|u_{m}\right\|_{W_{r}^{2,1}\left(\Omega^{T}\right)} \\
& \times\left(1+T^{(r-1) / r}\left\|u_{m}\right\|_{W_{r}^{2,1}\left(\Omega^{T}\right)}\right)\left\|u_{m+1}\right\|_{W_{r}^{2,1}\left(\Omega^{T}\right)}, \\
\|L\|_{W_{r}^{1,0}\left(\Omega^{T}\right)} \leq & \left\|\eta_{m}\left(\operatorname{div}-\operatorname{div}_{u_{m}}\right) u_{m+1}\right\|_{W_{r}^{1,0}\left(\Omega^{T}\right)} \\
\leq & c T^{(r-1) / r}\left\|u_{m}\right\|_{W_{r}^{2,1}\left(\Omega^{T}\right)}\left\|\eta_{m}\right\|_{V\left(\Omega^{T}\right)}\left\|u_{m+1}\right\|_{W_{r}^{2,1}\left(\Omega^{T}\right)} .
\end{aligned}
$$

Then by (3.5), and the estimates on $K$ and $L$, we obtain $X_{m+1} \leq c_{1} T^{(r-1) / r} X_{m}^{2} X_{m+1}+c_{2} T^{1 / r} X_{m} M+c_{3} T^{2(r-1) / r} X_{m}^{2} X_{m+1}+M$.

Since $r>3$ we have

$$
X_{m+1} \leq c_{4} T^{1 / r}\left(X_{m}+X_{m}^{2}\right) X_{m+1}+c_{5} T^{1 / r} X_{m} M+M .
$$

But we assume that $X_{m} \leq 4 M\left(X_{0}\right.$ satisfies (3.3)). Taking $T$ so small that

$$
c_{4} T^{1 / r}\left((4 M)+(4 M)^{2}\right)<1 / 2,
$$

we get

$$
X_{m+1} \leq 2 c_{5} T^{1 / r} X_{m} M+2 M
$$

and if $c_{5} T^{1 / r} 4 M<1$ we get $X_{m+1} \leq 4 M$. By induction we have proved that

$$
X_{k} \leq 4 M
$$

for all $k \in \mathbb{N}$. Finally, we choose $T^{*}$ so small that (1.7) is satisfied for all $\eta_{k}$. Let $U_{m}=u_{m+1}-u_{m}$ and $E_{m}=\eta_{m+1}-\eta_{m}$. Then from (3.4) we obtain

$$
\begin{array}{ll}
\eta_{m} U_{m, t}-\mu \Delta U_{m}-\nu \nabla \operatorname{div} U_{m}+p^{\prime}\left(\eta_{m}\right) \nabla E_{m}=M & \text { in } \Omega^{T}, \\
E_{m, t}+\eta_{m} \operatorname{div} U_{m}=N & \text { in } \Omega^{T}, \\
U_{m}=\alpha\left(x_{m}, t\right)-\alpha\left(x_{m-1}, t\right) & \text { on } S^{T}, \\
\left.U_{m}\right|_{t=0}=0,\left.\quad E_{m}\right|_{t=0}=0 & \text { on } \Omega,
\end{array}
$$


where

$$
\begin{aligned}
M= & -E_{m-1} u_{m, t}-\left(p^{\prime}\left(\eta_{m}\right)-p^{\prime}\left(\eta_{m-1}\right)\right) \nabla \eta_{m}+E_{m-1} f\left(x_{m}, t\right) \\
& +\left(f\left(x_{m}, t\right)-f\left(x_{m-1}, t\right)\right) \eta_{m-1} \\
& +\left(p^{\prime}\left(\eta_{m}\right)-p^{\prime}\left(\eta_{m-1}\right)\right)\left(\nabla-\nabla_{u_{m}}\right) \eta_{m+1} \\
& +p^{\prime}\left(\eta_{m}\right)\left(\nabla-\nabla_{u_{m}}\right) E_{m}+p^{\prime}\left(\eta_{m}\right)\left(\nabla_{u_{m}}-\nabla_{u_{m-1}}\right) \eta_{m} \\
& +\mu\left(\Delta_{u_{m}}-\Delta\right) U_{m}+\mu \operatorname{div}_{u_{m}}\left(\nabla_{u_{m}}-\nabla_{u_{m-1}}\right) u_{m} \\
& +\mu\left(\operatorname{div}_{u_{m}}-\operatorname{div}_{u_{m-1}}\right) \nabla_{u_{m-1}} u_{m}+\nu\left(\nabla_{u_{m}} \operatorname{div}_{u_{m}}-\nabla \operatorname{div}\right) U_{m} \\
& +\nu \nabla_{u_{m}}\left(\operatorname{div}_{u_{m}}-\operatorname{div}_{u_{m-1}}\right) u_{m}+\nu\left(\nabla_{u_{m}}-\nabla_{u_{m-1}}\right) \operatorname{div}_{u_{m-1}} u_{m}, \\
N= & E_{m-1}\left(\operatorname{div}-\operatorname{div}_{u_{m}}\right) u_{m+1}+\eta_{m-1}\left(\operatorname{div}-\operatorname{div}_{u_{m}}\right) U_{m} \\
& +\eta_{m-1}\left(\operatorname{div}_{u_{m-1}}-\operatorname{div}_{u_{m}}\right) u_{m}-E_{m-1} \operatorname{div}_{m} u_{m} .
\end{aligned}
$$

By Theorem 1.3 we get the estimate on $U_{m}$ and $E_{m}$ :

$$
\left\|U_{m}\right\|_{W_{r}^{2,1}\left(\Omega^{T}\right)}+\left\|E_{m}\right\|_{V\left(\Omega^{T}\right)}
$$$$
\leq c\left(\|M\|_{L_{r}\left(\Omega^{T}\right)}+\|N\|_{W_{r}^{1,0}\left(\Omega^{T}\right)}+\left\|\alpha\left(x_{m}, t\right)-\alpha\left(x_{m-1}, t\right)\right\|_{W_{r}^{2-1 / r, 1-1 /(2 r)}\left(S^{T}\right)}\right) .
$$

We have to estimate all the terms of the r.h.s. of (3.8). First we consider $M=\sum_{k=1}^{13} M_{k}$. By Proposition 2.2 and since $\left.E_{m-1}\right|_{t=0}=0$ we have

$$
\left\|M_{1}\right\|_{L_{r}\left(\Omega^{T}\right)}=\left\|E_{m-1} u_{m, t}\right\|_{L_{r}\left(\Omega^{T}\right)} \leq c T^{\alpha}\left\|E_{m-1}\right\|_{V^{\left(\Omega^{T}\right)}},
$$

and for $p^{\prime}(\cdot) \in C^{2}$,

$$
\begin{aligned}
\left\|M_{2}\right\|_{L_{r}\left(\Omega^{T}\right)} & =\left\|\left(p^{\prime}\left(\eta_{m}\right)-p^{\prime}\left(\eta_{m-1}\right)\right) \nabla \eta_{m}\right\|_{L_{r}\left(\Omega^{T}\right)} \leq c T^{\alpha}\left\|E_{m-1}\right\|_{V\left(\Omega^{T}\right)}, \\
\left\|M_{3}\right\|_{L_{r}\left(\Omega^{T}\right)} & =\left\|E_{m-1} f\left(x_{m}, t\right)\right\|_{L_{r}\left(\Omega^{T}\right)} \leq c T^{\alpha}\|f\|_{L_{r}\left(\Omega^{T}\right)}\left\|E_{m-1}\right\|_{V\left(\Omega^{T}\right)} .
\end{aligned}
$$

Since $f \in L_{r}\left(0, T ; W_{\infty}^{1}(\Omega)\right)$ we have

$$
\begin{aligned}
\left\|M_{4}\right\|_{L_{r}\left(\Omega^{T}\right)} & =\left\|\left(f\left(x_{m}, t\right)-f\left(x_{m-1}, t\right)\right) \eta_{m-1}\right\|_{L_{r}\left(\Omega^{T}\right)} \\
& \leq c T^{(r-1) / r}\left\|U_{m-1}\right\|_{W_{r}^{2,1}\left(\Omega^{T}\right)}, \\
\left\|M_{5}\right\|_{L_{r}\left(\Omega^{T}\right)} & =\left\|\left(p\left(\eta_{m}\right)-p^{\prime}\left(\eta_{m-1}\right)\right)\left(\nabla-\nabla_{u_{m}}\right) \eta_{m+1}\right\|_{L_{r}\left(\Omega^{T}\right)} \\
& \leq c T^{\alpha+(r-1) / r}\left\|E_{m-1}\right\|_{V\left(\Omega^{T}\right)}, \\
\left\|M_{6}\right\|_{L_{r}\left(\Omega^{T}\right)} & =\left\|p^{\prime}\left(\eta_{m}\right)\left(\nabla-\nabla_{u_{m}}\right) E_{m}\right\|_{L_{r}\left(\Omega^{T}\right)} \leq c T^{(r-1) / r}\left\|E_{m}\right\|_{V\left(\Omega^{T}\right)}, \\
\left\|M_{7}\right\|_{L_{r}\left(\Omega^{T}\right)} & =\left\|p^{\prime}\left(\eta_{m}\right)\left(\nabla_{u_{m}}-\nabla_{u_{m-1}}\right) \eta_{m}\right\|_{L_{r}\left(\Omega^{T}\right)} \\
& \leq c T^{(r-1) / r}\left\|U_{m-1}\right\|_{W_{r}^{2,1}\left(\Omega^{T}\right)} .
\end{aligned}
$$


As in the case of $K_{3}$ we estimate

$$
\begin{aligned}
\left\|M_{8}\right\|_{L_{r}\left(\Omega^{T}\right)} & =\left\|\mu\left(\Delta_{u_{m}}-\Delta\right) U_{m}\right\|_{L_{r}\left(\Omega^{T}\right)} \leq c T^{(r-1) / r}\left\|U_{m}\right\|_{W_{r}^{2,1}\left(\Omega^{T}\right)}, \\
\left\|M_{9}\right\|_{L_{r}\left(\Omega^{T}\right)} & =+\left\|\mu \operatorname{div}_{u_{m}}\left(\nabla_{u_{m}}-\nabla_{u_{m-1}}\right) u_{m}\right\|_{L_{r}\left(\Omega^{T}\right)}, \\
\left\|M_{10}\right\|_{L_{r}\left(\Omega^{T}\right)} & =\left\|\mu\left(\operatorname{div}_{u_{m}}-\operatorname{div}_{u_{m-1}}\right) \nabla_{u_{m-1}} u_{m}\right\|_{L_{r}\left(\Omega^{T}\right)} \\
& \leq c T^{(r-1) / r}\left\|U_{m-1}\right\|_{W_{r}^{2,1}\left(\Omega^{T}\right)} \\
\left\|M_{11}\right\|_{L_{r}\left(\Omega^{T}\right)} & =\left\|\nu\left(\nabla_{u_{m}} \operatorname{div}_{u_{m}}-\nabla \operatorname{div}\right) U_{m}\right\|_{L_{r}\left(\Omega^{T}\right)} \\
& \leq c T^{(r-1) / r}\left\|U_{m}\right\|_{W_{r}^{2,1}\left(\Omega^{T}\right)}, \\
\left\|M_{12}\right\|_{L_{r}\left(\Omega^{T}\right)} & =\left\|\nu \nabla_{u_{m}}\left(\operatorname{div}_{u_{m}}-\operatorname{div}_{u_{m-1}}\right) u_{m}\right\|_{L_{r}\left(\Omega^{T}\right)} \\
& \leq c T^{(r-1) / r}\left\|U_{m-1}\right\|_{W_{r}^{2,1}\left(\Omega^{T}\right)} \\
\left\|M_{13}\right\|_{L_{r}\left(\Omega^{T}\right)} & =\left\|\nu\left(\nabla_{u_{m}}-\nabla_{u_{m-1}}\right) \operatorname{div}_{u_{m-1}} u_{m}\right\|_{L_{r}\left(\Omega^{T}\right)} \\
& \leq c T^{(r-1) / r}\left\|U_{m-1}\right\|_{W_{r}^{2,1}\left(\Omega^{T}\right)}
\end{aligned}
$$

Now we estimate $N=\sum_{k=1}^{4} N_{k}$ :

$$
\begin{aligned}
\left\|N_{1}\right\|_{W_{r}^{1,0}\left(\Omega^{T}\right)} & =\left\|E_{m-1}\left(\operatorname{div}-\operatorname{div}_{u_{m}}\right) u_{m+1}\right\|_{W_{r}^{1,0}\left(\Omega^{T}\right)} \\
& \leq c T^{(r-1) / r}\left\|E_{m-1}\right\|_{V\left(\Omega^{T}\right)} \\
\left\|N_{2}\right\|_{W_{r}^{1,0}\left(\Omega^{T}\right)} & =\left\|\eta_{m-1}\left(\operatorname{div}-\operatorname{div}_{u_{m}}\right) U_{m}\right\|_{W_{r}^{1,0}\left(\Omega^{T}\right)} \\
& \leq c T^{(r-1) / r}\left\|U_{m}\right\|_{W_{r}^{2,1}\left(\Omega^{T}\right)} \\
\left\|N_{3}\right\|_{W_{r}^{1,0}\left(\Omega^{T}\right)} & =\left\|\eta_{m-1}\left(\operatorname{div}_{u_{m-1}}-\operatorname{div}_{u_{m}}\right) u_{m}\right\|_{W_{r}^{1,0}\left(\Omega^{T}\right)} \\
& \leq c T^{(r-1) / r}\left\|U_{m-1}\right\|_{W_{r}^{2,1}\left(\Omega^{T}\right)}, \\
\left\|N_{4}\right\|_{W_{r}^{1,0}\left(\Omega^{T}\right)} & =\left\|E_{m-1} \operatorname{div} u_{m}\right\|_{W_{r}^{1,0}\left(\Omega^{T}\right)} \\
& \leq c T^{\alpha}\left\|E_{m-1}\right\|_{V\left(\Omega^{T}\right)}+\left\|\nabla E_{m-1} \operatorname{div} u_{m}\right\|_{L_{r}\left(\Omega^{T}\right)} .
\end{aligned}
$$

To estimate the last term we note that $\operatorname{div} u_{m} \in W_{r}^{1,1 / 2}\left(\Omega^{T}\right)$ and

$$
\begin{aligned}
\left\|\operatorname{div} u_{m}\right\|_{L_{\infty}\left(\Omega^{T}\right)} & \leq c \sup _{t \leq T}\left\|\operatorname{div} u_{m}\right\|_{W_{r}^{1-2 / r}\left(\Omega^{T}\right)} \\
& \leq \widetilde{c}\left(\left\|u_{m}\right\|_{W_{r}^{2,1}\left(\Omega^{T}\right)}+\left\|u_{0}\right\|_{W_{r}^{2-2 / r}(\Omega)}\right),
\end{aligned}
$$

where $\widetilde{c}$ does not depend on $T$. Hence we get

$\left\|\nabla E_{m-1} \operatorname{div} u_{m}\right\|_{L_{r}\left(\Omega^{T}\right)}$

$$
\leq c\left\|\operatorname{div} u_{m}\right\|_{L_{\infty}\left(\Omega^{T}\right)}\left\|\int_{0}^{t}\left|\nabla E_{m-1, t}\right| d t^{\prime}\right\|_{L_{r}\left(\Omega^{T}\right)} \leq c T^{(r-1) / r}\left\|E_{m-1}\right\|_{V\left(\Omega^{T}\right)} .
$$

Thus

$$
\left\|N_{4}\right\|_{W_{r}^{1,0}\left(\Omega^{T}\right)} \leq c\left(T^{\alpha}+T^{(r-1) / r}\right)\left\|E_{m-1}\right\|_{V\left(\Omega^{T}\right)} .
$$


Now we estimate the r.h.s. of $(3.7)_{3}$. Since $\alpha \in C^{3}$ we have

$$
\left\|\alpha\left(x_{m}, t\right)-\alpha\left(x_{m-1}, t\right)\right\|_{W_{r}^{2-2 / r, 1-1 /(2 r)}\left(S^{T}\right)} \leq c T^{(r-1) / r}\left\|U_{m-1}\right\|_{W_{r}^{2,1}\left(\Omega^{T}\right)} .
$$

Let

$$
Y_{k}=\left\|U_{k}\right\|_{W_{r}^{2,1}\left(\Omega^{T}\right)}+\left\|E_{k}\right\|_{V\left(\Omega^{T}\right)} \text {. }
$$

Summing all estimates for the r.h.s. of (3.8) we obtain

$$
Y_{m} \leq c_{6} T^{\alpha} Y_{m-1}+c_{7} T^{(r-1) / r} Y_{m-1}+c_{8} Y_{m}
$$

Taking $T$ so small that $c_{8} T^{(r-1) / r}<1 / 2$, from (3.9) we get

$$
Y_{m} \leq c_{9}\left(T^{\alpha}+T^{(r-1) / r}\right) Y_{m-1}
$$

If $c_{9}\left(T^{\alpha}+T^{(r-1) / r}\right)<1$ we obtain $Y_{k} \rightarrow 0$ as $k \rightarrow \infty$.

Thus we have obtained (as $k \rightarrow \infty$ )

$$
\begin{array}{ll}
u_{k} \rightarrow u^{*} & \text { in } W_{r}^{2,1}\left(\Omega^{T}\right), \\
\eta_{k} \rightarrow \eta^{*} & \text { in } V\left(\Omega^{T}\right), \\
p^{\prime}\left(\eta_{k}\right) \rightarrow p^{\prime}\left(\eta^{*}\right) & \text { in } C^{\alpha}\left(\Omega^{T}\right) .
\end{array}
$$

We conclude that $\left(u^{*}, \eta^{*}\right)$ is a unique solution of (1.5) and the estimates (1.6) and (1.7) come from (3.6). We have obtained the local-in-time existence of solutions.

4. Almost global existence. First we show the boundedness of the $L_{2}$-norm of solutions of (1.9).

LEMMA 4.1. If $\|\sigma\|_{L_{\infty}(\Omega \times[0, t])}$ is sufficiently small then

$$
\|v(\cdot, t)\|_{L_{2}(\Omega)}+\|\sigma(\cdot, t)\|_{L_{2}(\Omega)} \leq A\left(\left\|v_{0}\right\|_{L_{2}(\Omega)}+\left\|\varrho_{0}-\bar{\varrho}\right\|_{L_{2}(\Omega)}\right),
$$

where $A$ is a positive constant.

Proof. Multiplying (1.1) 1 by $v$ and integrating over $\Omega$ we get

$$
\frac{d}{d t} \int_{\Omega}\left[\frac{1}{2} \varrho v^{2}+\frac{a}{\kappa-1} \varrho^{\kappa}-\varrho \varphi\right] d x+\int_{\Omega}\left[\mu|\nabla v|^{2}+\nu|\operatorname{div} v|^{2}\right] d x=0 .
$$

Next we examine

$$
I(\sigma)=\int_{\Omega}\left(\frac{a}{\kappa-1}(\bar{\varrho}+\sigma)^{\kappa}-(\bar{\varrho}+\sigma) \varphi\right) d x-\int_{\Omega}\left(\frac{a}{\kappa-1} \bar{\varrho}^{\kappa}-\bar{\varrho} \varphi\right) d x .
$$

It is easy to see that if $\|\sigma\|_{L_{\infty}}$ is sufficiently small then

$$
a_{1}\|\sigma\|_{L_{2}(\Omega)} \leq I(\sigma) \leq a_{2}\|\sigma\|_{L_{2}(\Omega)} .
$$

From (4.2) and (4.3) we obtain

$$
\frac{d}{d t}\left[\int_{\Omega} \frac{1}{2} \varrho v^{2} d x+I(\sigma)\right] \leq 0
$$

which gives (4.1) for $\sigma$ sufficiently small. 
Proof of Theorem 1.2. We examine (1.10) in the form

$$
\begin{aligned}
& \eta u_{t}-\mu \Delta u-\nu \nabla u+\gamma \nabla \chi=P, \\
& \chi_{t}+\eta \operatorname{div} u=Q, \\
& \left.u\right|_{S^{T}}=0, \\
& \left.u\right|_{t=0}=v_{0},\left.\quad \chi\right|_{t=0}=\sigma_{0},
\end{aligned}
$$

where

$$
\begin{aligned}
& P=\chi f-\chi \nabla_{u} \gamma-\mu\left(\Delta-\Delta_{u}\right) u-\nu\left(\nabla \operatorname{div} u-\nabla_{u} \operatorname{div}_{u}\right) u+\gamma\left(\nabla_{u}-\nabla\right) \chi \\
& Q=\eta\left(\operatorname{div}-\operatorname{div}_{u}\right) u-\bar{\eta}_{t} .
\end{aligned}
$$

By Theorem 1.3 we get the following estimate for solutions of (4.4):

$$
\begin{aligned}
& \|u\|_{W_{r}^{2,1}(\Omega \times[0, T])}+\|\eta\|_{V_{r}(\Omega \times[0, T])} \leq c\left(T, 4 \bar{\varrho}^{*}, \bar{\varrho}_{*} / 4\right) \\
& \times\left[\|P\|_{L_{r}(\Omega \times[0, T])}+\|Q\|_{W_{r}^{1,0}(\Omega \times[0, T])}+\left\|v_{0}\right\|_{W_{r}^{2-2 / r}(\Omega)}+\left\|\sigma_{0}\right\|_{W_{r}^{1}(\Omega)}\right] .
\end{aligned}
$$

From interpolation theorems we get

$$
\|\chi f\|_{L_{r}(\Omega \times[0, T])} \leq \varepsilon\|\chi\|_{V_{r}(\Omega \times[0, T])}+c(\varepsilon)\|\sigma\|_{L_{2}(\Omega \times[0, T])} .
$$

Since

$$
\|\chi\|_{L_{\infty}\left(0, T ; W_{r}^{1}(\Omega)\right)} \leq c\left(\|\chi\|_{V_{r}(\Omega \times[0, T])}+\left\|\sigma_{0}\right\|_{W_{r}^{1}(\Omega)}\right),
$$

we estimate

$$
\begin{aligned}
& \left\|\chi \nabla_{u} \gamma\right\|_{L_{r}(\Omega \times[0, T])} \\
\leq & c T^{1 / r}\left\|\sigma_{0}\right\|_{W_{r}^{1}(\Omega)}+c T^{1 / r} \varepsilon\|\chi\|_{V_{r}(\Omega \times[0, T])}+T^{1 / r} c(\varepsilon)\|\sigma\|_{L_{\infty}\left(0, T ; L_{2}(\Omega)\right)} .
\end{aligned}
$$

The rest is estimate by

$$
\begin{aligned}
& \left\|\left(\Delta-\Delta_{u}\right) u\right\|_{L_{r}(\Omega \times[0, T])} \leq c T^{(r-1) / r}\|u\|_{W_{r}^{2,1}(\Omega \times[0, T])}^{2}, \\
& \left\|\left(\nabla \operatorname{div} u-\nabla_{u} \operatorname{div}_{u}\right) u\right\|_{L_{r}(\Omega \times[0, T])} \leq c T^{(r-1) / r}\|u\|_{W_{r}^{2,1}(\Omega \times[0, T])}^{2}, \\
& \left\|\gamma\left(\nabla_{u}-\nabla\right) \chi\right\|_{L_{r}(\Omega \times[0, T])} \leq c T^{(r-1) / r}\|u\|_{W_{r}^{2,1}(\Omega \times[0, T])}\|\chi\|_{V_{r}(\Omega \times[0, T])}, \\
& \left\|\eta\left(\operatorname{div}-\operatorname{div}_{u}\right) u\right\|_{W_{r}^{1,0}(\Omega \times[0, T])} \leq c T^{(r-1) / r}\|u\|_{W_{r}^{2,1}(\Omega \times[0, T])}^{2} .
\end{aligned}
$$

Set

$$
\alpha=\|u\|_{W_{r}^{2,1}(\Omega \times[0, T])}+\|\chi\|_{V_{r}(\Omega \times[0, T])} .
$$

Taking $\varepsilon$ sufficiently small, from (4.5)-(4.8) we get

$$
\alpha \leq A \alpha^{2}+\beta,
$$

where

$$
A=c T^{(r-1) / r}, \quad \beta=c(\varepsilon, T)\left(\left\|v_{0}\right\|_{W_{r}^{2-2 / r}(\Omega)}+\left\|\sigma_{0}\right\|_{W_{r}^{1}(\Omega)}\right),
$$


and if the initial data are small enough (so that $1-4 A \beta>0$ ) then

$$
\alpha \leq \frac{1-\sqrt{1-4 A \beta}}{2 A} \leq 2 \beta .
$$

Estimate (4.9) gives the boundedness from Theorem 1.2. To end the proof of Theorem 1.2 we have to note that since we have (4.9), by Theorem 1.1 we can continue the solutions onto the whole interval $[0, T]$. Theorem 1.2 is proved.

\section{References}

[1] O. V. Besov, V. P. Il'in and S. M. Nikol'skiǔ, Integral Representations of Functions and Imbedding Theorems, Nauka, Moscow, 1975 (in Russian).

[2] A. Matsumura and T. Nishida, Initial boundary value problem for equations of motion of compressible viscous and heat conductive fluids, Comm. Math. Phys. 89 (1983), 445-464.

[3] P. B. Mucha and W. M. Zajączkowski, On the existence for the Dirichlet problem for the compressible linearized Navier-Stokes system in the $L_{p}$-framework, this issue, 000 .

[4] -, 一, Global existence of solutions of the Dirichlet problem for compressible NavierStokes equations, to appear.

[5] A. Valli and W. Zajacczkowski, Navier-Stokes equations for compressible fluids: global existence and qualitative properties of the solutions in the general case, Comm. Math. Phys. 103 (1986), 259-269.

Institute of Applied Mathematics and Mechanics

Warsaw University

Banacha 2

02-097 Warszawa, Poland

E-mail: mucha@hydra.mimuw.edu.pl
Institute of Mathematics Polish Academy of Sciences Śniadeckich 8 00-950 Warszawa, Poland E-mail: wz@impan.gov.pl

Institute of Mathematics and Operations Research Military University of Technology Kaliskiego 2 01-489 Warszawa, Poland 